\title{
Clinicopathological significance and prognostic value of Wilms' tumor gene expression in colorectal cancer
}

\author{
Yukinaga Miyata $^{\mathrm{a}, \mathrm{b}}$, Kenichi Kumagai ${ }^{\mathrm{a}, \mathrm{b}}$, Tomoko Nagaoka ${ }^{\mathrm{a}, \mathrm{b}}$, Kazutaka Kitaura ${ }^{\mathrm{b}}$, Goro Kaneda ${ }^{\mathrm{c}}$, \\ Hideki Kanazawa $^{\mathrm{c}}$, Satsuki Suzuki ${ }^{\mathrm{d}}$, Yoshiki Hamada ${ }^{\mathrm{a}}$ and Ryuji Suzukib,* \\ ${ }^{a}$ Department of Oral and Maxillofacial Surgery, School of Dental Medicine, Tsurumi University, Yokohama, Japan \\ ${ }^{\mathrm{b}}$ Department of Rheumatology and Clinical Immunology, Clinical Research Center for Rheumatology and Allergy, \\ Sagamihara National Hospital, National Hospital Organization, Sagamihara, Japan \\ ${ }^{\mathrm{c}}$ Department of Surgery Sagamihara National Hospital, National Hospital Organization, Sagamihara, Japan \\ ${ }^{\mathrm{d}}$ Section of Biological Science Research Center for Odontology, Nippon Dental University School of Dentistry, \\ Tokyo, Japan
}

\begin{abstract}
.
BACKGROUND: Colorectal cancer (CRC) is one of the most commonly diagnosed cancers, and novel effective treatments and diagnostic tools are urgently required.

OBJECTIVE: The selection of appropriate targeting tumor-associated antigens (TAAs) is critical for immunotherapy. Therefore, we analyzed TAA expression levels and investigated their relationship with clinical factors in adjacent normal mucosa (ANM) and CRC tissue.

METHODS: We obtained specimens of CRC primary tumors and matched ANM from 137 patients with CRC who underwent surgical resection. The mRNA levels of seven TAAs, Wilms' tumor gene (WT1), kinetochore associated-2 (KNTC2), cell division cycle associated-1 (CDCA1), M phase phosphoprotein-1 (MPHOSPH1), DEP domain-containing 1 (DEPDC1), 34-kDa translocase of the outer mitochondrial membrane (TOMM34) and ring finger protein-43 (RNF43), were analyzed using quantitative real-time reverse transcription-polymerase chain reaction, and their relationships with clinicopathological factors and the cell cycle were analyzed.

RESULTS: The expression levels of all seven TAAs were significantly higher in CRC tissues than in ANM. Expression levels of WT1 in CRC tissues did not correlate with the cell cycle. Furthermore, WT1 expression in CRC tissues was significantly related to tumor progression, lymph node metastasis, distant metastasis and clinical stage.

CONCLUSIONS: WT1 is a potential marker for prognosis and tumor progression in CRC.
\end{abstract}

Keywords: Colorectal cancer, tumor-associated antigens, Wilms' tumor gene

\footnotetext{
* Corresponding author: Ryuji Suzuki, Department of Rheumatology and Clinical Immunology, Clinical Research Center for Allergy and Rheumatology, Sagamihara National Hospital, National Hospital Organization, 18-1 Sakuradai, Minami-ku, Sagamihara, Kanagawa 252-0392, Japan. Tel.: +81 42742 8311; Fax: +81 42742 7990; E-mail: r-suzuki@sagamihara-hosp.gr.jp.
}

\section{Introduction}

Colorectal cancer (CRC) is one of the most commonly diagnosed cancers worldwide, and accounts for an estimated $9.4 \%$ of all malignancies [1]. In Asian countries, the incidence of CRC has increased approximately 2-4-fold over the past few decades [2]. The most effective treatment for CRC is surgery, but approximately $60 \%$ of patients who undergo curative re- 
section experience local recurrence or distant metastasis [3]. Despite advances in surgical techniques and chemotherapeutic options, the survival rate of CRC has not substantially improved; around $20 \%$ of patients with CRC die from recurrence of the disease [1]. Thus, novel effective treatments and diagnostic tools are required to treat CRC. New immunotherapies that target tumor-associated antigens (TAAs) have been used to treat $\mathrm{CRC}$, in addition to conventional treatments [4]. It has been demonstrated that CD8 cytotoxic T lymphocytes (CTLs) recognize epitope peptides derived from TAAs presented on MHC Class I molecules and then lyse the tumor cells. TAAs are proteins that are known to be overexpressed in and broadly distributed among malignant cells of various origins [5,6]. Many TAAs in various human malignancies were identified using cDNA expression cloning methods [7]. Immunotherapy is an active therapeutic approach designed to trigger the immune system to respond to tumor-specific antigens and attack tumor cells [8] and immunotherapy strategies include the use of peptides in TAAs. With antigenic epitope peptide vaccines derived from these TAAs, clinical trials have been initiated by multiple groups to treat cancer patients [9.-11]. Several vaccines for peptides, such as those for Wilms' tumor gene $(W T 1)$ [4 12], 34-kDa translocase of the outer mitochondrial membrane (TOMM34), and ring finger protein 43 (RNF43) [13] have been suggested to be clinically beneficial for metastatic CRC, in particular, TOMM34 and RNF43, which have been examined in phase I clinical trials [13]. These clinical trials have led to the possibility of developing new peptidebased cancer immunotherapies. However, these therapies have seen only a few recent successes and a tremendous number of failures [14]. In fact, clinical trials of TAA cell-targeted strategies are not always accepted because of the lack of reliable markers and the poor understanding of their behavior. However, combined immunotherapy and conventional chemotherapy treatments can simultaneously kill cancer cells and boost antitumor immune responses, and thus have significant advantages [15[16]. There have been reports that treatment with chemotherapy such as cyclophosphamide or gemcitabine can augment the antitumor effects of cancer immunotherapy [17], and immunotherapy has the potential to improve the effect of cancer treatments. Therefore, selection of appropriate targeting TAAs for effective immunotherapy is critical. An ideal TAA should have the characteristics of tumorspecific-antigens, which are silenced in normal tissues but overexpressed in cancer tissues. Immunotherapy is often recommended for advanced cancer patients in addition to surgery and chemotherapy, and thus it is preferable that the expression levels of TAAs are significantly higher in malignancies with tumor progression. The identification of TAAs at an early stage in tumor progression has clear implications for the continued development of cancer immunotherapy. Although TAA expression is generally associated with tumor progression and immunogenicity in various cancer types, little is known about their expression in relation to CRC progression [13]. In the present study, we analyzed TAA expression levels and investigated their relationship with clinical factors in ANM and CRC tissues using quantitative real-time reverse transcriptionpolymerase chain reaction (qPCR).

\section{Materials and methods}

\subsection{Patients and samples}

Tissue specimens were obtained from 137 patients (70 men, 67 women; age range, 30-91 years) who underwent surgical resection for CRC at Sagamihara National Hospital between 2010 and 2013. As control samples, resection specimens were taken from matched adjacent normal tissue (ANM) in CRC patients, and additional samples were taken from 10 patients with non-neoplastic colorectal polyps (CP). The ANM specimens were obtained from the margin of the CRC primary tumor, $\geqslant 10 \mathrm{~cm}$ away. All specimens were confirmed as CRC, ANM and CP by a pathologist. The ANM and CP specimens were further confirmed to be free of cancer cells by the pathologist. The diameters of the primary tumors $(\mathrm{T})$, numbers of lymph nodes with metastases $(\mathrm{N})$ and development of distant metastases (M) were classified according to the Union for International Cancer Control (UICC) TNM staging system. The study protocol was approved by the Research Ethical Committee of Sagamihara Hospital (Sagamihara, Japan). Informed consent for this study was obtained from all patients.

\subsection{Preparation of tissue specimens}

After surgery or biopsy, one-half of each CRC, ANM and CP specimen was immediately soaked in RNAlater RNA Stabilization Reagent (Qiagen, Hilden, Germany) and stored at $-80^{\circ} \mathrm{C}$ until RNA extraction.

\subsection{RNA extraction and cDNA synthesis}

CRC, ANM and CP specimens were homogenized with a vortex-type homogenizer (Shakeman 2; Bio 
Table 1

qPCR primer sequences

\begin{tabular}{lll}
\hline Targets & Forward primers $(5 /-3 \prime)$ & Reverse primers $(5 /-3 \prime)$ \\
\hline$G A P D H$ & GCACCGTCAAGGCTGAGAAC & ATGGTGGTGAAGACGCCAGT \\
$H P R T$ & GGCAGTATAATCCAAAGATGGTCAA & GTCAAGGGCATATCCTACAACAAAC \\
$A C T B$ & TGGCACCCAGCACAATGAA & CTAAGTCATAGTCCGCCTAGAAGCA \\
$S D H A$ & TGGGAACAAGAGGGCATCTG & CCACCACTGCATCAAATTCATG \\
$B 2 M$ & CTATCCAGCGTACTCCAAAGA & AAGACAAGTCTGAATGCTCCAC \\
$U B C$ & TCCCTTCTCGGCGATTCTG & TCTGCATTGTCATGAGGCGAT \\
$r R N A$ & CGACCATAAACGATGCCGAC & GGTGGTGCCCTTCCGTCAAT \\
$T B P$ & CCATGACTCCCGGAATCCCTAT & ATAGGCTGTGGGGTCAGTCCA \\
$W T 1$ & AGGGTACGAGCGATAACCACAC & CTCAGATGCCGACCGTACAAGA \\
$C D C A 1$ & AGAAGCATGCCGTGAAACGTA & CCATCTGAAAGCTGCTTGAACTC \\
$K N T C 2$ & AACCAAGGACCTGGAAGCTGAAC & TTTGGAATTCTCAGCACCTTTAGGA \\
$M P H O S P H 1$ & AATTGGTGTAAACCTGGCCACTA & CTTGCTTCTACATTTGAGAGCTTTG \\
$D E P D C 1$ & GGTTCTGATTATGCCTACTGGTTGA & TGGAATCTATCCATGTTCCAGCTTA \\
$T O M M 34$ & AAGCCAGAGTTCTGAAGGAAGAAGG & AGCTTGAGGGCTTCTGTGCAGT \\
$R N F 43$ & TGGTTACATCAGCATCGGACTTG & TGGAGTCTTCGACCTGGTTCTTG \\
$C Y C L I N A$ & CACTCTACACAGTCACGGGACAAAG & GTCTGGTGAAGGTCCATGAGACA \\
\hline
\end{tabular}

Medical Science, Tokyo, Japan). Total RNA was extracted using an RNeasy Lipid Tissue Kit (Qiagen). The quality and concentration of total RNA were validated using an Agilent 2100 Bioanalyzer (Agilent, Santa Clara, CA). Freshly isolated total RNA from CRC tissues and ANM was converted to cDNA using a PrimeScript RT Reagent Kit (Takara Bio, Shiga, Japan) according to the manufacturer's instructions.

\section{4. $q P C R$}

qPCR was performed using a Bio-Rad CFX96 system (Bio-Rad, Hercules, CA) following the manufacturer's protocols. Specific primers for WT1, cell division cycle associated 1 (CDCA1), kinetochore associated 2 (KNTC2), M phase phosphoprotein 1 (MPHOSPH1), DEP domain containing 1 (DEPDC1), TOMM34, RNF43 and cyclin A (CCNA2) and the housekeeping genes glyceraldehyde-3-phosphate dehydrogenase $(G A P D H)$, hypoxanthine phosphoribosyltransferase $(H P R T), \beta$-actin $(A C T B)$, succinate dehydrogenase complex, subunit A (SDHA), $\beta 2$-microgl ohulin $(B 2 M)$, ubiquitin $\mathrm{C}(U B C)$, $18 \mathrm{~S}$ ribosomal RNA ( $r R N A)$ and TATA-box binding protein (TBP) were purchased from Takara Bio (Otsu, Japan) (Table 1). The amplified products using the individual primer pairs were cloned into the pGEM-T vector (Promega, Madison, WI). The same plasmid was linearized by enzymatic digestion for use as a quantification standard. The sequences were confirmed by DNA sequencing using a CEQ8000 Genetic Analysis System (Beckman Coulter, Fullerton, CA). The quality and concentration of the quantification standard were validated using an Agilent 2100 Bioanalyzer (Agilent). The PCR reaction consisted of $5 \mu \mathrm{l}$ of SsoFast Eva-
Green Supermix (Bio-Rad), $3.5 \mu 1$ of RNase/DNasefree water, $0.5 \mu \mathrm{l}$ of $5 \mu \mathrm{M}$ primer mix, and $1 \mu \mathrm{l}$ of cDNA in a final volume of $10 \mu \mathrm{l}$. The cycle number for qPCR was 50. Calculated copy numbers were normalized based on the copy numbers of the housekeeping gene $A C T B$. All experiments were performed in duplicate.

\subsection{Statistical analyses}

To compare the results from the qPCR assays between expression levels in CRC, ANM and CP samples, we used one-way ANOVAs with Tukey-Kramer multiple comparison post-tests. Correlations between the expression levels of TAAs and CCNA2 used Spearman's rank coefficients. Correlations between TAA expression levels and clinical stages used Kruskal-Wallis tests and Pearson product-moment coefficients. Oneway ANOVAs with Tukey-Kramer multiple comparison post tests were performed for comparisons of the clinical stages. All statistical analyses were carried out using StatView software (SAS, Cary, NC). Results of comparisons are shown as mean values. $P$-values of $<$ 0.05 was considered significant.

\section{Results}

\subsection{Selection of appropriate housekeeping genes in CRC}

We investigated the mRNA levels of three housekeeping genes in CRC and ANM using qPCR (Fig. 1). $G A P D H, H P R T$ and $U B C$ mRNA levels were significantly higher in CRC tissues than in ANM $(P<$ $0.001)$, but $A C T B$ and $T B P$ were not significantly higher $(P=0.62$ and $P=0.55$, respectively). 


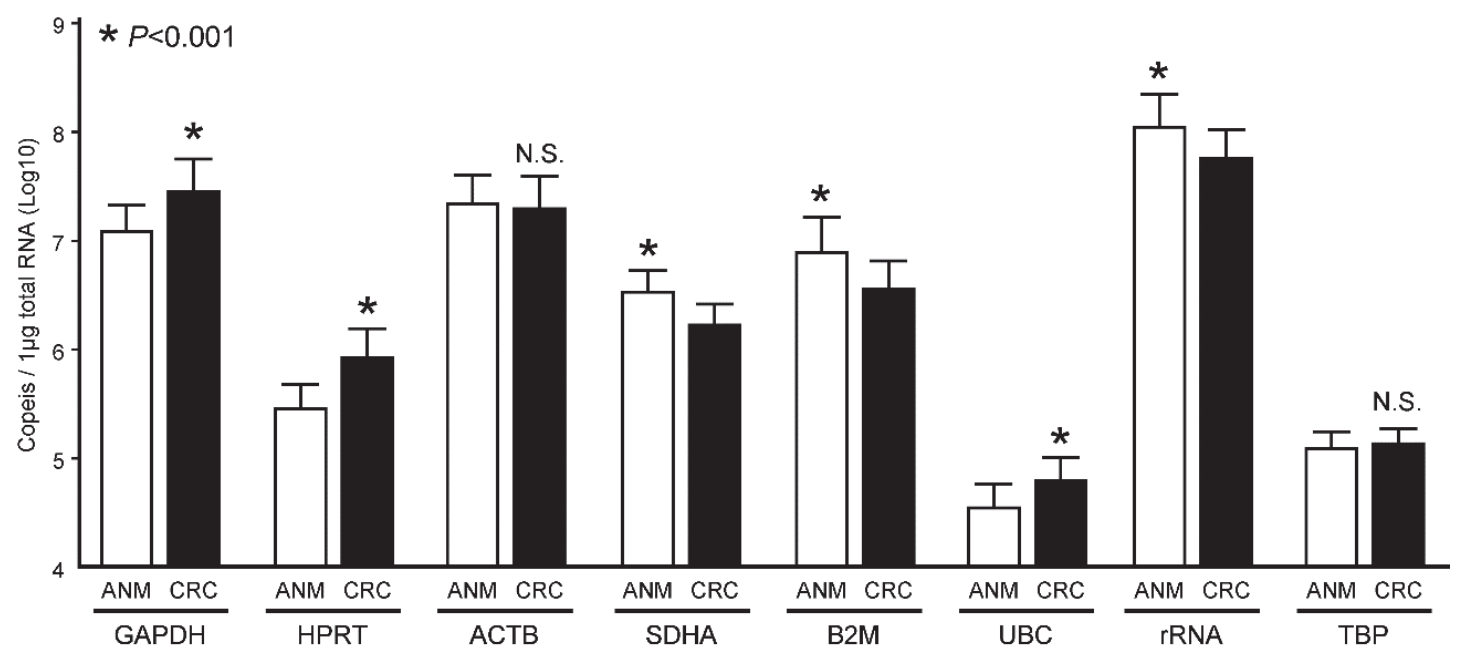

Fig. 1. Selection of appropriate housekeeping genes in CRC. mRNA expression levels of housekeeping genes were measured by qPCR in each tissue sample. Open and filled bars indicate ANM or CRC respectively; vertical error bars indicate mean \pm SD. The expression levels of $A C T B$ and TBP were almost equal in ANM and CRC tissues. Statistical analyses used Wilcoxon signed-rank tests. ${ }^{*} P<0.001$.

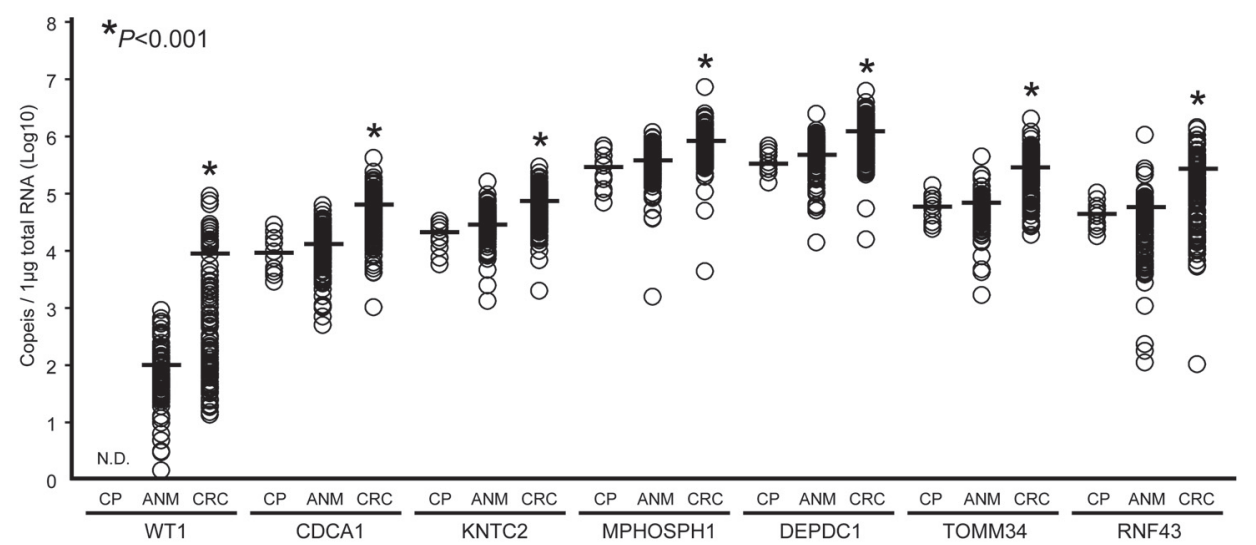

A

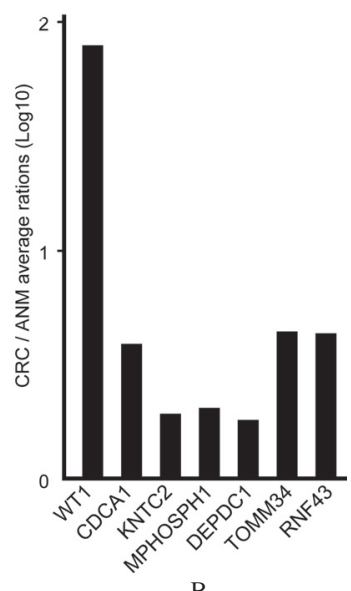

B

Fig. 2. Gene expression profiles of seven TAAs in ANM, CRC and CP tissues. Relative mRNA expression levels of TAAs in ANM, CRC and tissues were examined by qPCR. ACTB was used as an internal control. All experiments were performed in duplicate. (A) mRNA levels of all seven TAAs were significantly higher in CRC tissues than in ANM and CP samples. Statistical analyses used one-way ANOVAs with Tukey-Kramer multiple comparison post-tests. ${ }^{*} P<0.001$. (B) Comparisons of mRNA expression levels are shown as CRC/ANM average ratios. The mRNA expression of WT1 was increased by approximately 82 -fold, while other TAAs were increased around 2-5-fold.

\subsection{IRNA expression profiles of seven TAAs in NOM, ANM and CRC}

We next determined the mRNA levels of seven TAAs in 137 paired CRC tissues and ANM, and 10 CPs using qPCR. Expression levels of all seven TAAs were significantly increased in CRC tissues than in ANM and CP samples $(P<0.001$; Fig. 2A). In the ANM, the mRNA expression levels of WT1 were lower than other TAAs. The average expression level of WT1 was approximately 82-fold higher in CRC tissue than
ANM, whereas the other TAAs were only 2-5-fold higher (Fig. 2B). Moreover, in the CP samples, mRNA expression levels of WT1 were not detected.

\subsection{Correlations between TAA expression levels and CCNA2 in CRC}

We analyzed expression levels of CCNA2 and TAAs in CRC tissues (Fig. 3), using 44 randomly selected samples from the 137 samples. CCNA2 expression was strongly correlated with expression of $C D C A 1$, 

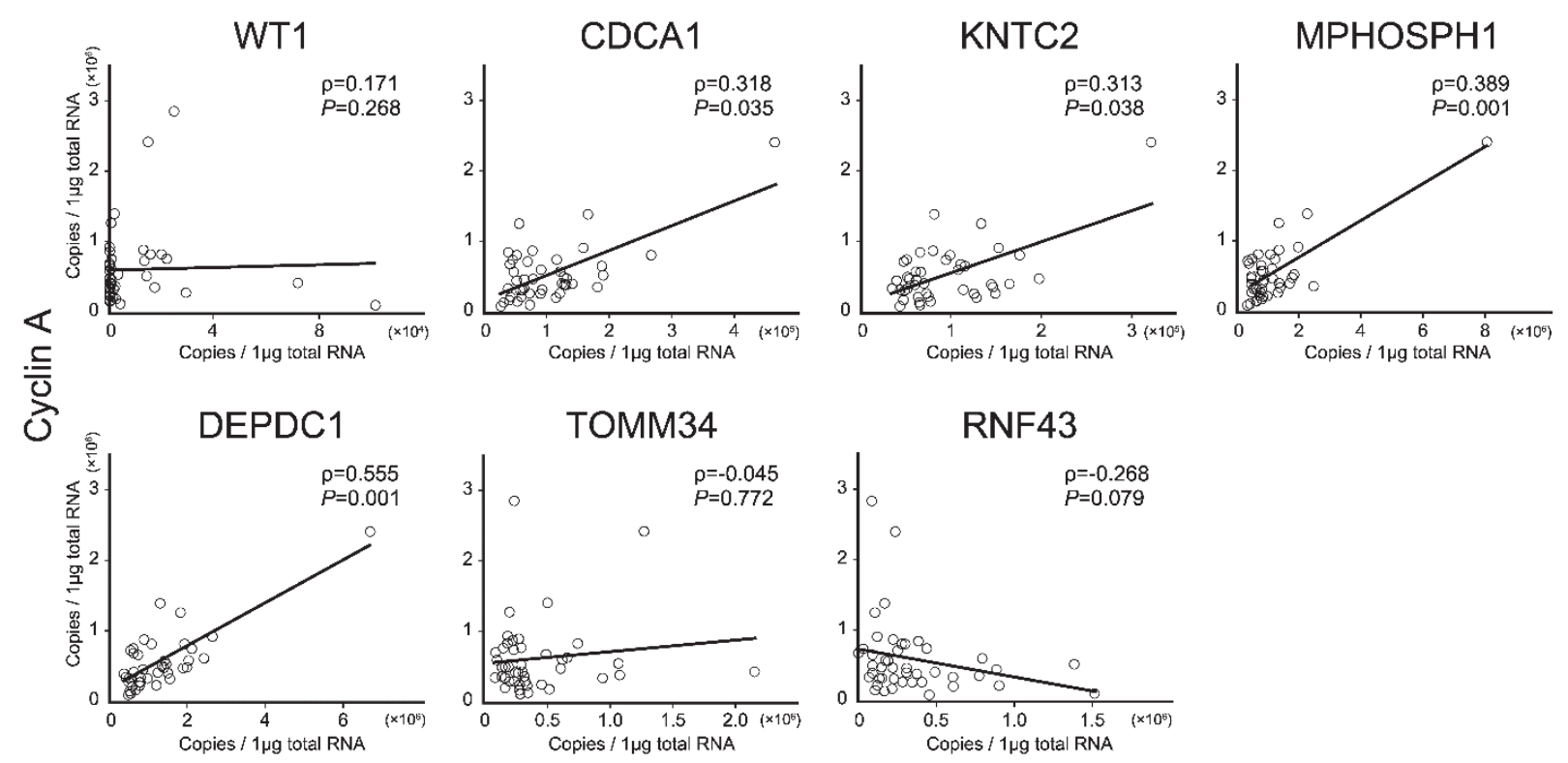

\section{TOMM34}

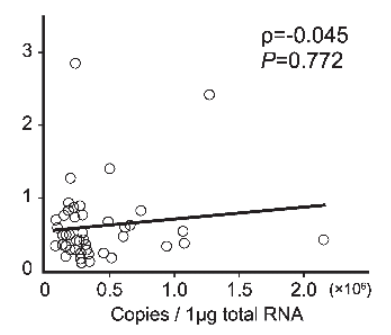

RNF43

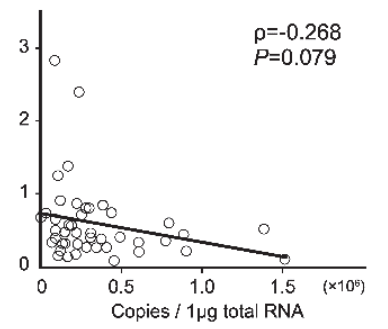

Fig. 3. Correlations between mRNA expression levels of TAAs and CCNA2 in CRC. The mRNA expression levels of TAAs and CCNA2 were measured by qPCR. ACTB was used as an internal control. Expression levels of $C C N A 2$ in CRC tissues significantly correlated with $C D C A 1$, KNTC2, MPHOSPH1, and DEPDC1 in CRC tissues, but not with WT1, TOMM34 and RNF43. Statistical analyses used Spearman's rank correlation coefficient.

KNTC2, MPHOSPH1 and DEPDCl in CRC tissues $(P=0.035, P=0.038, P=0.001$, and $P=$ 0.001 , respectively), but not with the expression of WT1, TOMM34 and RNF43 $(P=0.268, P=0.772$ and $P=0.079$, respectively).

\subsection{Clinicopathological factors and expression levels of WT1, TOMM34 and RNF43 in CRC}

Next, we analyzed clinicopathological factors and WT1, TOMM34, and RNF43 levels in CRC tissues (Table 2). Specimens of advanced $\mathrm{T}$ stage had significantly greater $W T 1$ expression $(P=0.039)$, but not TOMM34 and RNF43, than early-stage specimens. Similarly, specimens with lymph node metastasis and distant metastasis had significantly greater WT1 expression than negative specimens $(P=0.023$ and $P=$ 0.036 , respectively), but not TOMM34 and RNF43. Moreover, fifteen of the $137 \mathrm{CRC}$ patients who underwent surgery died within 5 years, and the specimens from these patients had greater WT1 expression than those from patients who remained alive, but there was no significant difference $(P=0.259)$. TOMM34 and RNF43 were also found to not be significant.

We also found significant differences between WT1 mRNA expression levels and clinical stages in CRC tissues ( $P=0.011$; Table 2$)$, but not TOMM34, and
RNF43. Clinical stages were correlated highly with WT1 expression levels in CRC $(r=0.2541 ; P=$ 0.002; Fig. 4). However, we found no significant associations between expression of WT1, TOMM34 and RNF43 in terms of sex, age or site of tumor (Table 2).

\section{Discussion}

In the present study, we analyzed the mRNA expression levels of seven TAAs in specimens from 137 CRC patients. First, we selected an appropriate housekeeping gene to use as a standard in our analysis. Among the eight known housekeeping genes, GAPDH is considered to be an inappropriate internal standard gene for cancer because of the hypoxic conditions in cancer tissues [18]; Zhong et al. reported that GAPDH mRNA expression levels in cell lines are increased by 21.2-75.1\% under hypoxic conditions [19]. Jacques et al. also reported that the coefficients of correlation between expression levels of $T B P$ in colon samples and CRC had quite a positive result in their experiment [20]. Another report suggested that $A C T B$ is the most stable gene in diabetic glomeruli and primary mesangial cells [21]. Surprisingly, in our study, the expression levels of $A C T B$ and $T B P$ were almost equal in ANM and CRC tissues. Therefore, ACTB was se- 
Table 2

Comparative analyses of clinicopathological characteristics and WT1, TOMM34, and RNF43 status in patients with CRC

\begin{tabular}{|c|c|c|c|c|c|}
\hline \multicolumn{2}{|c|}{ Clinicopathological characteristics } & \multirow{2}{*}{$\begin{array}{l}\text { Number of patients }(\%) \\
137(100.0)\end{array}$} & \multirow[t]{2}{*}{$W T 1^{(c)}$} & \multirow[t]{2}{*}{$\operatorname{TOMM} 4^{(c)}$} & \multirow[t]{2}{*}{$R N F 43^{(c)}$} \\
\hline All cases & & & & & \\
\hline Gender $^{(a)}$ & $\begin{array}{l}\text { Male } \\
\text { Female }\end{array}$ & $\begin{array}{l}67(48.9) \\
70(51.1)\end{array}$ & 0.815 & 0.865 & 0.672 \\
\hline $\operatorname{Age}^{(a)}$ & $\begin{array}{l}<65 \text { years } \\
\geqslant 65 \text { years }\end{array}$ & $\begin{array}{l}40(29.2) \\
97(70.8)\end{array}$ & 0.998 & 0.446 & 0.935 \\
\hline Site of tumors ${ }^{(b)}$ & $\begin{array}{l}\text { Rectum } \\
\text { Sigmoid colon } \\
\text { Descending colon } \\
\text { Transverse colon } \\
\text { Ascending colon } \\
\text { Cecum }\end{array}$ & $\begin{aligned} 32 & (23.4) \\
42 & (30.7) \\
8 & (5.8) \\
11 & (8.0) \\
34 & (24.8) \\
10 & (7.3)\end{aligned}$ & 0.144 & 0.067 & 0.906 \\
\hline Survival $^{(a)}$ & $\begin{array}{l}\text { Survival } \\
\text { Death }\end{array}$ & $\begin{array}{r}122(89.1) \\
15(10.9)\end{array}$ & 0.259 & 0.567 & 0.691 \\
\hline T stage ${ }^{(a)}$ & $\begin{array}{l}\mathrm{pT} 1 / \mathrm{T} 2 \\
\mathrm{pT} 3 / \mathrm{T} 4\end{array}$ & $\begin{array}{r}21(15.3) \\
116(84.7)\end{array}$ & 0.039 & 0.723 & 0.799 \\
\hline $\mathrm{N}$ stage ${ }^{(a)}$ & $\begin{array}{l}\mathrm{pN} 0 \\
\mathrm{pN} 1-\mathrm{N} 3\end{array}$ & $\begin{array}{l}57(41.6) \\
80(58.4)\end{array}$ & 0.023 & 0.831 & 0.081 \\
\hline M stage ${ }^{(a)}$ & $\begin{array}{l}\text { M0 } \\
\text { M1 }\end{array}$ & $\begin{array}{r}115(83.9) \\
22(16.1)\end{array}$ & 0.036 & 0.503 & 0.997 \\
\hline Clinical stage ${ }^{(b)}$ & $\begin{array}{l}\text { I } \\
\text { II } \\
\text { III } \\
\text { IV }\end{array}$ & $\begin{array}{l}16(11.7) \\
41(29.9) \\
58(42.3) \\
22(16.1)\end{array}$ & 0.011 & 0.926 & 0.345 \\
\hline
\end{tabular}

${ }^{(a)}$ Mann-Whitney U test; (b) Kruskal-Wallis test; (c) $P$-values; significant differences are in boldface.

lected as the internal standard gene in the present study. Moreover, we analyzed $T B P$ as an internal control to obtain reliable results. The calculated copy numbers were normalized based on the copy numbers of $T B P$ and an analogous result was obtained using $A C T B$. We found that, although the mRNA expression levels of all seven TAAs were significantly greater in CRC tissues than in ANM, mRNA expression of WT1 in CRC tissue was approximately 82-fold higher than in ANM.

Expression of TAAs is restricted to a limited set of tissues such as the fetal kidney, ovary, testis, spleen, and mesothelial cell lining of visceral organs [56]. However, in the present study, TAA expression was also detected in the ANM tissues. Although TAAs that decorate cancer cells are targets for the immune system, many are not truly cancer-specific because they can also be found on normal tissues [22]. This arises through the presence of pre-malignant cells that express TAAs in normal-appearing colorectal mucosal tissues. This concept is field cancerization, or the field effect, which was first described by Slaughter et al. in oral carcinoma [23]. The concept of field cancerization implies that tumor carcinogenesis may even occur in the ANM. A previous study showed that gene expression in ANM cells in CRC patients was not quite normal, and this may have important implications for CRC prognosis and progression [22]. Moreover, in the present study, mRNA expression levels of WT1 were not detected in the CP samples. It is thought that WT1 has strong tissue specificity in CRC [12]. In selecting appropriate TAAs, their expression in adjacent tissues is important. Ideal TAAs for peptide vaccines would show strongly specific expression and correlation with clinicopathological factors. In fact, the correlation of WT1 mRNA with CRC pathological conditions strengthens the clinical significance of WT1 in CRC.

Regarding the cell cycle, we investigated the correlations between TAAs and a cyclin family member. Cell proliferation follows an orderly progression through the cell cycle, which is governed by protein complexes composed of cyclins such as cyclins A, B1, B2, C, D1-3, and E and cyclin-dependent kinases [24]. Cyclin A achieves its maximal level during the $S$ and $G_{2}$ phases, and is thus regarded as a regulator of the transition to mitosis; it may directly affect the entry into mitosis of cancer cells, and therefore mediate the high proliferative activity of cancer cells [25]. The cell cycle of cancer cells is faster than that of normal cells, and the expression level of cyclin $\mathrm{A}$ is known to be increased in cancer cells [26]. We investigated the association between TAA expression levels and cyclin A (CCNA2), and revealed some close relationships in $\mathrm{CRC}$ tissues. Identification of TAAs that are not af- 

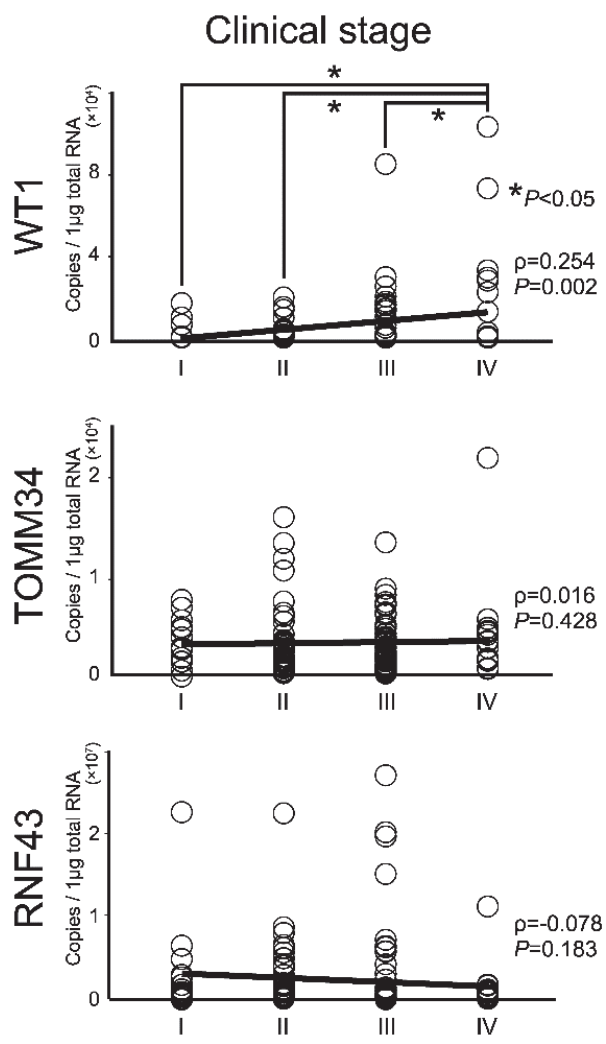

Fig. 4. Correlations among expression levels of WT1, TOMM34 and RNF43 and CRC clinical stage. mRNA expression levels of WT1, TOMM34, and RNF43 and clinical stage were evaluated in CRC tissue. WT1 mRNA expression correlated only with clinical stage $(r=$ 0.254; $P=0.002)$. Statistical analyses used the Pearson product-moment correlation and one-way ANOVAs with Tukey-Kramer multiple comparison post-tests. ${ }^{*} P<0.05$.

fected by cell proliferation was an important consideration. In the present study, we found no correlations between CCNA2 and WT1, TOMM34, and RNF43 in $\mathrm{CRC}$ tissues. Among the CRC clinicopathological factors, only WT1 showed a significant association with lymph node metastasis, distant metastasis, T-stage, or clinical stage, whereas the other TAAs did not. Therefore, WT1 could be an appropriate TAA marker for tumor progression in CRC.

WT1 was originally isolated as a tumor-suppressor gene that is inactivated in a subset of Wilms' tumors and mutated in the germline of children with a genetic predisposition to this kidney neoplasm in childhood [27-29]. The wild-type WT1 protein was shown to be expressed in cancer cells derived from various kinds of cancers, including colon cancer [12], and overexpressed in primary leukemia [30], breast cancer [31|32], lung cancer [33], bone and soft-tissue sarcoma [34], and head and neck squamous cell carci- noma [35]. Recently, a National Cancer Institute pilot project to prioritize cancer antigens made efforts to develop both a priority-ranked list of known cancer antigens, and a list of weighted "ideal" antigen criteria/ characteristics for evaluating TAAs [36]. Interestingly, WT1 headed this list, supporting translational research for further design of WT1-based cancer vaccines [36].

The mRNA expression levels of WT1 in CRC correlated with distant metastasis, clinical stage, and lymph node metastasis. Additionally, fifteen of the $137 \mathrm{CRC}$ patients who underwent surgery died within 5 years, and the specimens from these patients had greater WT1 expression than those from patients who remained alive, but there was no significant difference. Our collection of CRC tissue specimens was examined between 2010 and 2013, therefore, we could not analyze the 5-year survival rate. We will continue the investigation of survival rates in the future. However, we did find that the expression of WT1 in CRC tissues was significantly related to tumor progression, lymph node metastasis, distant metastasis and clinical stage, thus WT1 expression may be correlated with survival rate. Moreover, Oji et al. [12] reported that WT1 mRNA expression in CRC was significantly higher than that in normal-appearing colorectal mucosal tissues, with no significant correlations between WT1 mRNA expression and clinical factors and TNM stages. Although our results were not consistent with this report, the number of samples in our study was much larger, and therefore WT1 expression may reflect tumor progression and metastatic activity. These analyses of WT1 will increase our understanding of TAAs in CRC pathogenesis, and could further lead to novel treatment and early diagnostic tools and offer new strategies for TAAbased immunotherapies in CRC.

\section{Conclusions}

WT1 is an appropriate TAA for CRC, and WT1 mRNA expression in CRC primary tumors could be a novel independent marker for prognosis and tumor progression.

\section{Acknowledgements}

This study was supported by Chugai Pharmaceutical Company Limited, Takeda Pharmaceutical Company Limited and Yakult Honsha Company Limited. 


\section{References}

[1] R. Siegel, J. Ma, Z. Zou and A. Jemal, Cancer statistics, 2014, CA Cancer J Clin 64 (2014), 9-29.

[2] J.J. Sung, J.Y. Lau, K.L. Goh and W.K. Leung, Increasing incidence of colorectal cancer in Asia: implications for screening, Lancet Oncol 6 (2005), 871-876.

[3] N. Andre and W. Schmiegel, Chemoradiotherapy for colorectal cancer, Gut 54 (2005), 1194-1202.

[4] Y. Oka, A. Tsuboi, T. Taguchi, T. Osaki, T. Kyo, H. Nakajima, O.A. Elisseeva, Y. Oji, M. Kawakami, K. Ikegame, N. Hosen, S. Yoshihara, F. Wu, F. Fujiki, M. Murakami, T. Masuda, S. Nishida, T. Shirakata, S. Nakatsuka, A. Sasaki, K. Udaka, H. Dohy, K. Aozasa, S. Noguchi, I. Kawase and H. Sugiyama, Induction of WT1 (Wilms' tumor gene)-specific cytotoxic T lymphocytes by WT1 peptide vaccine and the resultant cancer regression, Proc Natl Acad Sci U S A 101 (2004), 1388513890 .

[5] J. Pelletier, M. Schalling, A.J. Buckler, A. Rogers, D.A. Haber and D. Housman, Expression of the Wilms' tumor gene WT1 in the murine urogenital system, Genes Dev 5 (1991), 13451356.

[6] A. Huang, C.E. Campbell, L. Bonetta, M.S. McAndrewsHill, S. Chilton-MacNeill, M.J. Coppes, D.J. Law, A.P. Feinberg, H. Yeger and B.R. Williams, Tissue, developmental, and tumor-specific expression of divergent transcripts in Wilms tumor, Science 250 (1990), 991-994.

[7] M. Ito, S. Shichijo, N. Tsuda, M. Ochi, N. Harashima, N. Saito and K. Itoh, Molecular basis of T cell-mediated recognition of pancreatic cancer cells, Cancer Res 61 (2001), 20382046.

[8] S. Koido, T. Ohkusa, S. Homma, Y. Namiki, K. Takakura, K. Saito, Z. Ito, H. Kobayashi, M. Kajihara, K. Uchiyama, S. Arihiro, H. Arakawa, M. Okamoto, J. Gong and H. Tajiri, Immunotherapy for colorectal cancer, World J Gastroenterol 19 (2013), 8531-8542.

[9] S.A. Rosenberg, J.C. Yang, D.J. Schwartzentruber, P. Hwu, F.M. Marincola, S.L. Topalian, N.P. Restifo, M.E. Dudley, S.L. Schwarz, P.J. Spiess, J.R. Wunderlich, M.R. Parkhurst, Y. Kawakami, C.A. Seipp, J.H. Einhorn and D.E. White, Immunologic and therapeutic evaluation of a synthetic peptide vaccine for the treatment of patients with metastatic melanoma, Nat Med 4 (1998), 321-327.

[10] B. Mukherji, N.G. Chakraborty, S. Yamasaki, T. Okino, H. Yamase, J.R. Sporn, S.K. Kurtzman, M.T. Ergin, J. Ozols and $\mathbf{J}$ Meehan, Induction of antigen-specific cytolytic $\mathrm{T}$ cells in situ in human melanoma by immunization with synthetic peptide-pulsed autologous antigen presenting cells, Proc Natl Acad Sci USA 92 (1995), 8078-8082.

[11] X. Hu, N.G. Chakraborty, J.R. Sporn, S.H. Kurtzman, M.T. Ergin and Mukherji B, Enhancement of cytolytic T lymphocyte precursor frequency in melanoma patients following immunization with the MAGE-1 peptide loaded antigen presenting cell-based vaccine, Cancer Res 56 (1996), 2479-2483

[12] Y. Oji, H. Yamamoto, M. Nomura, Y. Nakano, A. Ikeba, S. Nakatsuka, S. Abeno, E. Kiyotoh, T. Jomgeow, M. Sekimoto, R. Nezu, Y. Yoshikawa, Y. Inoue, N. Hosen, M. Kawakami, A. Tsuboi, Y. Oka, H. Ogawa, S. Souda, K. Aozasa, M. Monden and H. Sugiyama, Overexpression of the Wilms' tumor gene WT1 in colorectal adenocarcinoma, Cancer Sci 94 (2003), 712-717.

[13] K. Okuno, F. Sugiura, J.I. Hida, T. Tokoro, E. Ishimaru, Y. Sukegawa and K. Ueda, Phase I clinical trial of a novel pep- tide vaccine in combination with UFT/LV for metastatic colorectal cancer, Exp Ther Med 2 (2011), 73-79.

[14] A. Kudrin and M.G. Hanna, Jr., Overview of the cancer vaccine field: are we moving forward? Hum Vaccin Immunother 8 (2012), 1135-1140.

[15] R.A. Lake and B.W. Robinson, Immunotherapy and chemotherapy - a practical partnership. Nat Rev Cancer 5 (2005), 397-405.

[16] A.K. Nowak, B.W. Robinson and R.A. Lake, Synergy between chemotherapy and immunotherapy in the treatment of established murine solid tumors. Cancer Res 63 (2003), 44904496.

[17] S. Kan, S. Hazama, K. Maeda, Y. Inoue, S. Homma, S. Koido, M. Okamoto and M. Oka, Suppressive effects of cyclophosphamide and gemcitabine on regulatory T-cell induction in vitro. Anticancer Res 32 (2012), 5363-5369.

[18] J. Caradec, N. Sirab, D. Revaud, C. Keumeugni and S. Loric, Is GAPDH a relevant housekeeping gene for normalisation in colorectal cancer experiments? Br J Cancer 103 (2010), 1475-1476.

[19] H. Zhong and JW. Simons, Direct comparison of GAPDH, beta-actin, cyclophilin, and 28S rRNA as internal standards for quantifying RNA levels under hypoxia Biochem Biophys Res Commun 259 (1999), 523-526.

[20] J.B. Kok, R.W. Roelofs, B.A. Giesendorf, J.L. Pennings, E.T. Waas, T. Feuth, D.W. Swinkels and P.N. Span. Normalization of gene expression measurements in tumor tissues: Comparison of 13 endogenous control genes. Lab Invest 85 (2005), 154-159.

[21] J. Biederman, J. Yee and P. Cortes, Validation of internal control genes for gene expression analysis in diabetic glomerulosclerosis. Kidney Int 66 (2004), 2308-2314.

[22] R. Sanz-Pamplona, A. Berenguer, D. Cordero, D.G. Mollevi, M. Crous-Bou, X. Sole, L. Pare-Brunet, E. Guino, R. Salazar, C. Santos, J. de Oca, X. Sanjuan, F. Rodriguez-Moranta and V. Moreno, Aberrant gene expression in mucosa adjacent to tumor reveals a molecular crosstalk in colon cancer, $\mathrm{Mol}$ Cancer 13 (2014), 46.

[23] D.P. Slaughter, H.W. Southwick and W. Smejkal, Field cancerization in oral stratified squamous epithelium; clinical implications of multicentric origin, Cancer 6 (1953), 963-968.

[24] M. Lotayef, G.D. Wilson, F.M. Daley, H.K. Awwad and T. Shouman, Aberrant expression of cyclin A in head and neck cancer, Br J Cancer 83 (2000), 30-34.

[25] C. Cordon-Cardo, Mutations of cell cycle regulators. Biological and clinical implications for human neoplasia, Am J Pathol 147 (1995), 545-560.

[26] K. Handa, M. Yamakawa, H. Takeda, S. Kimura and T. Takahashi, Expression of cell cycle markers in colorectal carcinoma: superiority of cyclin A as an indicator of poor prognosis, Int J Cancer 84 (1999), 225-233.

[27] E.M. Algar, M.T. Kenney, L.A. Simms, S.I. Smith, Y. Kida and P.J. Smith, Homozygous intragenic deletion in the WT1 gene in a sporadic Wilms' tumour associated with high levels of expression of a truncated transcript, Hum Mutat 5 (1995), 221-227.

[28] K.M. Call, T. Glaser, C.Y. Ito, A.J. Buckler, J. Pelletier, D.A. Haber, E.A. Rose, A. Kral, H. Yeger, W.H. Lewis and et al., Isolation and characterization of a zinc finger polypeptide gene at the human chromosome 11 Wilms' tumor locus, Cell 60 (1990), 509-520.

[29] J. Pelletier, W. Bruening, F.P. Li, D.A. Haber, T. Glaser and D.E. Housman, WT1 mutations contribute to abnormal gen- 
ital system development and hereditary Wilms' tumour, $\mathrm{Na}$ ture 353 (1991), 431-434.

[30] K. Inoue, H. Sugiyama, H. Ogawa, M. Nakagawa, T. Yamagami, H. Miwa, K. Kita, A. Hiraoka, T. Masaoka, K. Nasu and et al., WT1 as a new prognostic factor and a new marker for the detection of minimal residual disease in acute leukemia, Blood 84 (1994), 3071-3079.

[31] D.M. Loeb, E. Evron, C.B. Patel, P.M. Sharma, B. Niranjan, L. Buluwela, S.A. Weitzman, D. Korz and S. Sukumar, Wilms' tumor suppressor gene (WT1) is expressed in primary breast tumors despite tumor-specific promoter methylation, Cancer Res 61 (2001), 921-925.

[32] Y. Miyoshi, A. Ando, C. Egawa, T. Taguchi, Y. Tamaki, H. Tamaki, H. Sugiyama and S. Noguchi, High expression of Wilms' tumor suppressor gene predicts poor prognosis in breast cancer patients, Clin Cancer Res 8 (2002), 1167-1171.

[33] Y. Oji, S. Miyoshi, H. Maeda, S. Hayashi, H. Tamaki, S. Nakatsuka, M. Yao, E. Takahashi, Y. Nakano, H. Hirabayashi, Y. Shintani, Y. Oka, A. Tsuboi, N. Hosen, M. Asada, T. Fujioka, M. Murakami, K. Kanato, M. Motomura, E.H. Kim, M. Kawakami, K. Ikegame, H. Ogawa, K. Aozasa, I. Kawase and H. Sugiyama, Overexpression of the Wilms' tumor gene WT1 in de novo lung cancers, Int J Cancer 100 (2002), 297303.

[34] T. Ueda, Y. Oji, N. Naka, Y. Nakano, E. Takahashi, S. Koga, M. Asada, A. Ikeba, S. Nakatsuka, S. Abeno, N. Hosen, Y. Tomita, K. Aozasa, N. Tamai, A. Myoui, H. Yoshikawa and H. Sugiyama, Overexpression of the Wilms' tumor gene WT1 in human bone and soft-tissue sarcomas, Cancer Sci 94 (2003), 271-276.

[35] Y. Oji, H. Inohara, M. Nakazawa, Y. Nakano, S. Akahani, S. Nakatsuka, S. Koga, A. Ikeba, S. Abeno, Y. Honjo, Y. Yamamoto, S. Iwai, K. Yoshida, Y. Oka, H. Ogawa, J. Yoshida, K. Aozasa, T. Kubo and H. Sugiyama, Overexpression of the Wilms' tumor gene WT1 in head and neck squamous cell carcinoma, Cancer Sci 94 (2003), 523-529.

[36] M.A. Cheever, J.P. Allison, A.S. Ferris, O.J. Finn, B.M. Hastings, T.T. Hecht, I. Mellman, S.A. Prindiville, J.L. Viner, L.M. Weiner and L.M. Matrisian, The prioritization of cancer antigens: A national cancer institute pilot project for the acceleration of translational research, Clin Cancer Res 15 (2009), 5323-5337. 